\title{
Strong Negative Type in Spheres
}

\author{
By Russell LyOns
}

\begin{abstract}
It is known that spheres have negative type, but only subsets with at most one pair of antipodal points have strict negative type. These are conditions on the (angular) distances within any finite subset of points. We show that subsets with at most one pair of antipodal points have strong negative type, a condition on every probability distribution of points. This implies that the function of expected distances to points determines uniquely the probability measure on such a set. It also implies that the distance covariance test for stochastic independence, introduced by Székely, Rizzo and Bakirov, is consistent against all alternatives in such sets. Similarly, it allows tests of goodness of fit, equality of distributions, and hierarchical clustering with angular distances. We prove this by showing an analogue of the Cramér-Wold theorem.
\end{abstract}

\section{$\S 1$. Introduction.}

We introduce the topic by borrowing from Lyons (2014).

Let $(X, d)$ be a metric space. One says that $(X, d)$ has negative type if for all $n \geq 1$ and all lists of $n$ red points $x_{i}$ and $n$ blue points $x_{i}^{\prime}$ in $X$, the sum $2 \sum_{i, j} d\left(x_{i}, x_{j}^{\prime}\right)$ of the distances between the $2 n^{2}$ ordered pairs of points of opposite color is at least the sum $\sum_{i, j}\left(d\left(x_{i}, x_{j}\right)+d\left(x_{i}^{\prime}, x_{j}^{\prime}\right)\right)$ of the distances between the $2 n^{2}$ ordered pairs of points of the same color. It is not obvious that euclidean space has this property, but it is well known. By considering repetitions of $x_{i}$ and taking limits, we arrive at a superficially more general property: For all $n \geq 1, x_{1}, \ldots, x_{n} \in X$, and $\alpha_{1}, \ldots, \alpha_{n} \in \mathbb{R}$ with $\sum_{i=1}^{n} \alpha_{i}=0$, we have

$$
\sum_{i, j \leq n} \alpha_{i} \alpha_{j} d\left(x_{i}, x_{j}\right) \leq 0 .
$$

We say that $(X, d)$ has strict negative type if, for every $n$ and all $n$-tuples of distinct points $x_{1}, \ldots, x_{n}$, equality holds in (1.1) only when $\alpha_{i}=0$ for all $i$. Again, euclidean spaces have strict negative type. A simple example of a metric space of non-strict negative type is $\ell^{1}$ on a 2 -point space, i.e., $\mathbb{R}^{2}$ with the $\ell^{1}$-metric.

A (Borel) probability measure $\mu$ on $X$ has finite first moment if $\int d(o, x) \mathrm{d} \mu(x)<\infty$ for some (hence all) $o \in X$; write $P_{1}(X, d)$ for the set of such probability measures. Suppose that $\mu_{1}, \mu_{2} \in P_{1}(X, d)$. By approximating $\mu_{i}$ by probability measures of finite support, we obtain a yet more general property, namely, that when $X$ has negative type,

$$
\int d\left(x_{1}, x_{2}\right) \mathrm{d}\left(\mu_{1}-\mu_{2}\right)^{2}\left(x_{1}, x_{2}\right) \leq 0 .
$$

We say that $(X, d)$ has strong negative type if it has negative type and equality holds in (1.2) only when $\mu_{1}=\mu_{2}$. See Lyons (2018) for an example of a (countable) metric space of strict but not strong negative

2010 Mathematics Subject Classification. Primary 51K99, 51M10, 45Q05, 44A12. Secondary 62H20, 62G20, 62H15, 62H30. Key words and phrases. Cramér-Wold, hemispheres, expected distances, distance covariance, equality of distributions, goodness of fit, hierarchical clustering.

Research partially supported by NSF grant DMS-1612363. 
type. The notion of strong negative type was first defined by Zinger, Kakosyan, and Klebanov 1992). Lyons (2013) used it to show that a metric space $X$ has strong negative type iff the theory of distance covariance holds in $X$ just as in euclidean spaces, as introduced by Székely, Rizzo, and Bakirov (2007). Similarly, it allows tests of goodness of fit, equality of distributions, and hierarchical clustering with angular distances: see the review in Székely and Rizzo (2017). Lyons (2013) noted that if $(X, d)$ has negative type, then $\left(X, d^{r}\right)$ has strong negative type when $0<r<1$.

Define

$$
a_{\mu}(x):=\int d\left(x, x^{\prime}\right) \mathrm{d} \mu\left(x^{\prime}\right)
$$

for $x \in X$ and $\mu \in P_{1}(X, d)$. Lyons (2013) remarked that if $(X, d)$ has negative type, then the map $\alpha: \mu \mapsto a_{\mu}$ is injective on $\mu \in P_{1}(X)$ iff $X$ has strong negative type. (There are also metric spaces not of negative type for which $\alpha$ is injective.)

A list of metric spaces of negative type appears as Theorem 3.6 of Meckes (2013). All euclidean spaces have strong negative type; see Lyons (2013) for a discussion of various proofs.

That real and complex hyperbolic spaces $\mathbb{H}^{n}$ have negative type was shown by Gangolli (1967), Sec. 4, and was made explicit by Faraut and Harzallah (1974), Corollary 7.4; that they have strict negative type was shown by Hjorth, Kokkendorff, and Markvorsen (2002). Lyons (2014) showed that real hyperbolic spaces have strong negative type. The remaining constant-curvature, simply connected spaces are spheres.

Let $S^{n}$ denote the unit-radius sphere centered at the origin of $\mathbb{R}^{n+1}$. Although spheres have negative type (in their intrinsic metric), not even circles have strict negative type. For example, in $S^{1}$, take two red points $\{(1,0),(-1,0)\}$ and two blue points $\{(0,1),(0,-1)\}$. Nevertheless, antipodal symmetry is the only obstruction to strict negative type: the main result, Theorem 9.1, of Hjorth, Lisoněk, Markvorsen, and Thomassen (1998) is that a subset of a sphere has strict negative type iff that subset contains at most one pair of antipodal points. We strengthen this to strong negative type:

THEOREM 1.1. If $B \subset S^{n}$ contains at most one pair of antipodal points, then $B$ has strong negative type.

We begin by proving a special case:

THEOREM 1.2. If $H \subset S^{n}$ is an open hemisphere, then $H$ has strong negative type.

We may parametrize open hemispheres as

$$
H_{t}:=\left\{x \in S^{n} ; t \cdot x>0\right\}
$$

for $t \in S^{n}$. A crucial ingredient in the proof of Theorem 1.2 is an analogue of the Cramér-Wold theorem:

TheOREM 1.3. Let $H$ be an open hemisphere in an $n$-dimensional sphere, $S^{n}$. For a finite signed measure $\mu$ on $H$ and $t \in S^{n}$, define $b_{\mu}(t):=\mu\left(H \cap H_{t}\right)$. The map $\mu \mapsto b_{\mu}$ is injective. Moreover, if $D$ is a dense subset of $S^{n}$, then $\mu \mapsto b_{\mu}\lceil D$ is injective.

Let $R: x \mapsto-x$ be the reflection in the origin. If $K$ is a Borel subset of $S^{n}$ such that $K$ and its image under $R$ partition $S^{n}$ and such that the interior of $K$ is a hemisphere, then call $K$ a partitioning hemisphere. Given a probability measure $\mu$ on $S^{n}$, let $\mu_{R}$ denote the maximal measure that is invariant under $R$ and such that $\mu_{R} \leq \mu$. Note that if $\mu$ is a probability measure on $S^{n}$ that is invariant under $R$, then $\mu(K)=1 / 2$ for every partitioning hemisphere, $K$. Therefore, for every probability measure $\mu$ on $S^{n}$ with $\mu_{R} \neq 0$, there is a probability measure $\nu \neq \mu$ such that $\mu(K)=\nu(K)$ for every partitioning hemisphere, $K$. Moreover, if $\mu_{R} \neq 0$ but $\mu_{R}(A)=0$ for every $(n-1)$-dimensional great sphere $A$ in $S^{n}$, then there is another 
probability measure $\nu \neq \mu$ such that $\mu(H)=\nu(H)$ for every open hemisphere, $H$. We extend Theorem 1.3 to show the converse, which we use to prove Theorem 1.1:

THEOREM 1.4. Let $\mu$ be a probability measure on $S^{n}$ such that $\mu_{R}=0$. If $\nu$ is a probability on $S^{n}$ such that $\mu(K)=\nu(K)$ for every partitioning hemisphere, $K$, then $\nu=\mu$. Similarly, if $\nu$ is a probability on $S^{n}$ such that $\mu\left(H_{t}\right)=\nu\left(H_{t}\right)$ for every $t$ belonging to a dense subset $D$ of $S^{n}$, then $\nu=\mu$.

The last assertion of Theorem 1.4 is essentially known: see, e.g., Lemmas 2.3 and 2.4 of Rubin (1999).

We also have the following fact:

Proposition 1.5. If $\mu$ is a probability measure on $S^{n}$ such that $\mu(K)=1 / 2$ for every partitioning hemisphere, $K$, then $\mu$ is $R$-invariant. Similarly, if $\mu$ is a probability measure on $S^{n}$ such that $\mu\left(H_{t}\right)=\mu\left(H_{-t}\right)$ for a dense set of $t$, then $\mu$ is $R$-invariant.

The last assertion is again essentially known: see Korollar 3.2 of Schneider (1970). The work of Rubin (1999) and Schneider (1970), as well as other authors who study related questions, uses spherical harmonics. This is a powerful tool that leads to more general results, although those extensions do not seem relevant to negative type. We give elementary proofs that rely only on the Cramér-Wold theorem for euclidean spaces:

THEOREM 1.6. If $\mu$ is a complex Borel measure on $\mathbb{R}^{n}$ such that $\mu(H)=0$ for every open halfspace in $\mathbb{R}^{n}$, then $\mu=0$.

Proof. For $t \in S^{n-1}$, define $\mu_{t}$ on $\mathbb{R}$ by

$$
\mu_{t}(-\infty, a):=\mu\left\{x \in \mathbb{R}^{n} ; t \cdot x<a\right\} \quad(a \in \mathbb{R})
$$

Then $\mu_{t}=0$, whence its Fourier transform $\widehat{\mu_{t}}$ satisfies $\widehat{\mu_{t}}(b)=0$ for all $b \in \mathbb{R}$. Because $\widehat{\mu_{t}}(b)=\widehat{\mu}(b t)$, it follows that the Fourier transform of $\mu$ also vanishes, whence so does $\mu$.

Even this theorem can be proved without Fourier analysis - see Walther 1997) or Lyons and Zumbrun (2018).

\section{$\S$ 2. Proofs.}

Proof of Theorem 1.9. By the bounded convergence theorem, $b_{\mu}\left\lceil D\right.$ determines $b_{\mu}(t)$ for all $t$ such that $\mu\left(\partial\left(H \cap H_{t}\right)\right)=0$, and therefore $b_{\mu}\left\lceil D\right.$ determines all of $b_{\mu}$ by continuity from below: for every $t \in S^{n}$, there are $s_{k} \in S^{n}$ such that $\mu\left(\partial\left(H \cap H_{s_{k}}\right)\right)=0$ and $H \cap H_{s_{k}}$ increase to $H \cap H_{t}$.

We may take $H$ to be the upper open hemisphere, $\left\{\left(t_{1}, t_{2}, \ldots, t_{n+1}\right) \in S^{n} ; t_{n+1}>0\right\}$. Define $\phi: H \rightarrow$ $\mathbb{R}^{n+1}$ by

$$
\phi\left(t_{1}, \ldots, t_{n+1}\right):=\left(t_{1} / t_{n+1}, \ldots, t_{n} / t_{n+1}, 1\right) .
$$

Then $\phi$ is a homeomorphism from $H$ to the affine hyperplane $H^{\prime}:=\left\{\left(t_{1}, t_{2}, \ldots, t_{n+1}\right) \in \mathbb{R}^{n+1} ; t_{n+1}>0\right\}$, namely, $\phi(t)$ is the intersection of $H^{\prime}$ with the line through the origin and $t$. Furthermore, $\phi$ maps $H \cap H_{t}$ to an open halfspace in $H^{\prime}$ and every open halfspace in $H^{\prime}$ is the image under $\phi$ of some $H \cap H_{t}$. Therefore, $b_{\mu}$ determines the measures of all open halfspaces with respect to the pushforward $\phi_{*} \mu$ on $H^{\prime}$. The classical theorem of Cramér and Wold applied to $H^{\prime}$ shows that this determines $\phi_{*} \mu$, which in turn determines $\mu$. 
Proof of Theorem 1.2. Write $\sigma$ for the volume measure on $S^{n}$ normalized to have mass $\pi$. Then for all $x_{1}, x_{2} \in S^{n}$, we have

$$
d\left(x_{1}, x_{2}\right)=\int\left|\mathbf{1}_{H_{t}}\left(x_{1}\right)-\mathbf{1}_{H_{t}}\left(x_{2}\right)\right|^{2} \mathrm{~d} \sigma(t)
$$

This well-known fact is easy to see: By rotation-invariance of $\sigma$, the right-hand side depends only on $d\left(x_{1}, x_{2}\right)$. By considering three points on a great circle, we find that the dependence is linear. Finally, by taking antipodal points, we verify that the constant of linearity is 1 .

Therefore, if $\mu_{1}$ and $\mu_{2}$ are probabilities on $S^{n}$, we may write

$$
\int d\left(x_{1}, x_{2}\right) \mathrm{d}\left(\mu_{1}-\mu_{2}\right)^{2}\left(x_{1}, x_{2}\right)=\iint\left|\mathbf{1}_{H_{t}}\left(x_{1}\right)-\mathbf{1}_{H_{t}}\left(x_{2}\right)\right|^{2} \mathrm{~d}\left(\mu_{1}-\mu_{2}\right)^{2}\left(x_{1}, x_{2}\right) \mathrm{d} \sigma(t) .
$$

Expanding the square in the integrand and using the facts that

$$
\int \mathbf{1}_{H_{t}}(x) \mathrm{d} \nu^{2}(x, y)=\nu\left(H_{t}\right) \nu\left(S^{n}\right)
$$

and

$$
\int \mathbf{1}_{H_{t}}(x) \mathbf{1}_{H_{t}}(y) \mathrm{d} \nu^{2}(x, y)=\nu\left(H_{t}\right)^{2}
$$

for any finite signed measure, $\nu$, we obtain that

$$
\int d\left(x_{1}, x_{2}\right) \mathrm{d}\left(\mu_{1}-\mu_{2}\right)^{2}\left(x_{1}, x_{2}\right)=-2 \int\left(\mu_{1}\left(H_{t}\right)-\mu_{2}\left(H_{t}\right)\right)^{2} \mathrm{~d} \sigma(t) .
$$

It is evident from this that $\left(S^{n}, d\right)$ has negative type. In order to prove $(H, d)$ has strong negative type, it suffices to show that if $\mu_{1}$ and $\mu_{2}$ are concentrated on $H$ and satisfy $\mu_{1}\left(H_{t}\right)=\mu_{2}\left(H_{t}\right)$ for $\sigma$-a.e. $t$, then $\mu_{1}=\mu_{2}$. But this is immediate from Theorem 1.3.

Given any signed measure $\theta$, define the antisymmetric measure $\bar{\theta}:=\theta-R_{*} \theta$, where $R_{*} \theta$ is the pushforward of $\theta$ by $R$. For positive $\theta$ with $\theta_{R}=0$, we have $\theta=\bar{\theta}^{+}$, the positive part of $\bar{\theta}$. For positive $\theta$ without assuming that $\theta_{R}=0$, we have

$$
2 \theta\left(S^{n}\right) \geq|\bar{\theta}|\left(S^{n}\right), \text { with equality iff } \theta_{R}=0 .
$$

LEMMA 2.1. Let $\mu$ and $\nu$ be probability measures on $S^{n}$. If $\mu(K)=\nu(K)$ for every partitioning hemisphere, $K$, then $\bar{\mu}=\bar{\nu}$. Similarly, if $\mu\left(H_{t}\right)=\nu\left(H_{t}\right)$ for every $t \in D$, where $D$ is a dense subset of $S^{n}$, then $\bar{\mu}=\bar{\nu}$.

Proof. We claim that there is an $(n-1)$-dimensional great sphere $A$ in $S^{n}$ with $\mu(A)=\nu(A)=0$. To see this, we build $A$ inductively by dimension. First, because only countably many points have positive mass, there is a pair $A_{0}$ of antipodal points with $\mu\left(A_{0}\right)=\nu\left(A_{0}\right)=0$. Second, all uncountably many 1-dimensional great spheres in $S^{n}$ that contain $A_{0}$ have pairwise intersections exactly $A_{0}$, whence there is a 1-dimensional great sphere $A_{1} \supset A_{0}$ with $\mu\left(A_{1}\right)=\nu\left(A_{1}\right)=0$. We may continue this procedure recursively, finding a $k$-dimensional great sphere $A_{k} \supset A_{k-1}$ for $1 \leq k \leq n-1$ with $\mu\left(A_{k}\right)=\nu\left(A_{k}\right)=0$. Finally, take $A:=A_{n-1}$.

Let $H$ be one of the two open hemispheres comprising $S^{n} \backslash A$. Note that $\mu(H)=\nu(H)$ under either assumption (in the second case, we use a continuity argument like that at the start of the proof of Theorem 1.3.

Let $K$ be a partitioning hemisphere. Because $\mu(A)=0$ and $\mu\left(S^{n}\right)=1$, we have

$$
\bar{\mu}(H \cap K)=\mu(H \cap K)+\mu(H \cap R K)-\mu(H \cap R K)-\mu(R H \cap R K)=\mu(H)-\mu(R K)=\mu(H)+\mu(K)-1 .
$$


A similar equation holds for $\nu$. Hence, the assumption that $\mu(K)=\nu(K)$ for every partitioning hemisphere, $K$, yields

$$
\bar{\mu}(H \cap K)=\bar{\nu}(H \cap K)
$$

for every such $K$.

Now every set $H \cap H_{t}$ is of the form $H \cap K$ for some partitioning hemisphere, $K$. It follows that

$$
\bar{\mu}\left(H \cap H_{t}\right)=\bar{\nu}\left(H \cap H_{t}\right)
$$

for every $t$, whence by Theorem 1.3 , it follows that $\bar{\mu}=\bar{\nu}$.

We now prove the second assertion of the lemma. Note that in the preceding proof, we did not use the full strength of the assumption that $\mu(K)=\nu(K)$ for every partitioning hemisphere, $K$, but only that $\mu\left(K_{t}\right)=\nu\left(K_{t}\right)$ for partitioning hemispheres $K_{t}$ satisfying $K_{t} \cap H=H_{t} \cap H$ for $t \in D$; we may also require that $K_{t} \cap R H=\overline{H_{t}} \cap R H$. Let $u$ be such that $H_{u}=H$, and let $s_{k}$ be on the geodesic segment from $u$ to $t$ with $s_{k} \notin\{u, t\}, s_{k} \rightarrow t$, and $\mu\left(\partial H_{s_{k}}\right)=\nu\left(\partial H_{s_{k}}\right)=0$. (Such $s_{k}$ exist because $\mu(A)=\nu(A)=0$.) Let $t_{k, j} \in D$ converge to $s_{k}$ as $j \rightarrow \infty$. By the bounded convergence theorem, $\lim _{j \rightarrow \infty} \mu\left(H_{t_{k, j}}\right)=\mu\left(H_{s_{k}}\right)$ and similarly for $\nu$, whence $\mu\left(H_{s_{k}}\right)=\nu\left(H_{s_{k}}\right)$. In addition, we have $\lim _{k \rightarrow \infty} \mu\left(H_{s_{k}}\right)=\mu\left(K_{t}\right)$ and similarly for $\nu$. Hence, $\mu\left(K_{t}\right)=\nu\left(K_{t}\right)$ for every $t \in D$, whence $\bar{\mu}=\bar{\nu}$.

Proof of Theorem 1.4. By Lemma 2.1, either assumption implies that $\bar{\mu}=\bar{\nu}$. We may conclude from (2.1) that $2=2 \nu\left(S^{n}\right) \geq|\bar{\nu}|\left(S^{n}\right)=|\bar{\mu}|\left(S^{n}\right)=2 \mu\left(S^{n}\right)=2$, whence again from [2.1), that $\nu_{R}=0$. Since also $\mu_{R}=0$, we obtain the desired conclusion, $\mu=\bar{\mu}^{+}=\bar{\nu}^{+}=\nu$.

Proof of Theorem 1.1. If $B$ contains no antipodal points, then every $\mu$ concentrated on $B$ has $\mu_{R}=0$, whence the proof that $B$ has strong negative type is exactly as for Theorem 1.2, using Theorem 1.4 in place of Theorem 1.3.

If $B$ contains one antipodal pair, $\{x, R x\}$, then it still suffices to show that for probabilities $\mu$ and $\nu$ concentrated on $B$, the assumption $\mu\left(H_{t}\right)=\nu\left(H_{t}\right)$ for a dense set of $t$ implies $\mu=\nu$. By Lemma 2.1, such an assumption yields $\bar{\mu}=\bar{\nu}$. Because $\bar{\mu}=\overline{\mu-\mu_{R}}$ and $\mu-\mu_{R}$ is a positive measure with $\left(\mu-\mu_{R}\right)_{R}=0$, and similarly for $\nu$, we obtain $\overline{\mu-\mu_{R}}=\overline{\nu-\nu_{R}}$ and $\mu-\mu_{R}={\overline{\mu-\mu_{R}}}^{+}=\overline{\nu-\nu_{R}}+=\nu-\nu_{R}$. Therefore, $\mu_{R}\left(H_{t}\right)=\nu_{R}\left(H_{t}\right)$ for a dense set of $t$. Because $\mu_{R}$ and $\nu_{R}$ are supported by $\{x, R x\}$, it follows that $\mu_{R}=\nu_{R}$, and so $\mu=\nu$, as desired.

Proof of Proposition 1.5. For both assertions, we may apply Lemma 2.1 to the pair of measures $\mu$ and $R_{*} \mu$, getting $\bar{\mu}=-\bar{\mu}$, whence $\bar{\mu}=0$. Thus, $\mu=R_{*} \mu$, as desired.

Acknowledgement. I thank Marcos Matabuena for asking me about strong negative type for the angular metric on compositional data, i.e., on the probability simplex. 


\section{REFERENCES}

Faraut, J. and Harzallah, K. (1974). Distances hilbertiennes invariantes sur un espace homogène. Ann. Inst. Fourier (Grenoble), 24(3), xiv, 171-217.

Gangolli, R. (1967). Positive definite kernels on homogeneous spaces and certain stochastic processes related to Lévy's Brownian motion of several parameters. Ann. Inst. H. Poincaré Sect. B (N.S.), 3, $121-226$.

Hjorth, P., Lisoněk, P., Markvorsen, S., and Thomassen, C. (1998). Finite metric spaces of strictly negative type. Linear Algebra Appl., 270, 255-273.

Hjorth, P.G., Kokkendorff, S.L., and Markvorsen, S. (2002). Hyperbolic spaces are of strictly negative type. Proc. Amer. Math. Soc., 130(1), 175-181 (electronic).

Lyons, R. (2013). Distance covariance in metric spaces. Ann. Probab., 41(5), 3284-3305.

Lyons, R. (2014). Hyperbolic space has strong negative type. Illinois J. Math., 58(4), 1009-1013.

Lyons, R. (2018). Errata to "Distance covariance in metric spaces" [MR3127883]. Ann. Probab., 46(4), $2400-2405$.

Lyons, R. and Zumbrun, K. (2018). A calculus proof of the Cramér-Wold theorem. Proc. Amer. Math. Soc., 146(3), 1331-1334.

Meckes, M.W. (2013). Positive definite metric spaces. Positivity, 17(3), 733-757.

Rubin, B. (1999). Inversion and characterization of the hemispherical transform. J. Anal. Math., 77, $105-128$.

SCHNEIDER, R. (1970). Über eine Integralgleichung in der Theorie der konvexen Körper. Math. Nachr., 44, $55-75$.

SzÉKely, G.J. and Rizzo, M.L. (2017). The energy of data. Annu. Rev. Stat. Appl., 4, 447-479.

SzÉKely, G.J., Rizzo, M.L., and BAKIrov, N.K. (2007). Measuring and testing dependence by correlation of distances. Ann. Statist., 35(6), 2769-2794.

Walther, G. (1997). On a conjecture concerning a theorem of Cramér and Wold. J. Multivariate Anal., 63(2), 313-319. Addendum, J. Multivariate Anal. 67(2) (1998), 431.

Zinger, A.A., Kakosyan, A.V., and Klebanov, L.B. (1992). A characterization of distributions by mean values of statistics and certain probabilistic metrics. J. Soviet Math., 59(4), 914-920. Stability problems for stochastic models. Translated from Problemy Ustoichivosti Stokhasticheskikh Modelei, Trudy Seminara, 1989, pp. 47-55.

Department of Mathematics, 831 E. 3Rd St., Indiana University, Bloomington, IN 47405-7106

rdlyonsoindiana.edu

http://pages.iu.edu/"rdlyons/ 